\title{
Effect of Heat and Mass Transfer and Magnetic Field on Peristaltic Flow of a Fractional Maxwell Fluid in a Tube
}

\author{
F. S. Bayones, ${ }^{1}$ A. M. Abd-Alla $\left(\mathbb{D},{ }^{2}\right.$ and Esraa N. Thabet ${ }^{2}{ }^{2}$ \\ ${ }^{1}$ Department of Mathematics and Statistics, College of Science, Taif University, P.O. Box 11099, Taif 21944, Saudi Arabia \\ ${ }^{2}$ Department of Mathematics, Faculty of Science, Sohag University, Sohag, Egypt \\ Correspondence should be addressed to A. M. Abd-Alla; mohmrr@yahoo.com
}

Received 20 March 2021; Revised 28 April 2021; Accepted 31 May 2021; Published 17 June 2021

Academic Editor: Ahmed Mostafa Khalil

Copyright (c) 2021 F. S. Bayones et al. This is an open access article distributed under the Creative Commons Attribution License, which permits unrestricted use, distribution, and reproduction in any medium, provided the original work is properly cited.

Magnetic field and the fractional Maxwell fluids' impacts on peristaltic flows within a circular cylinder tube with heat and mass transfer were evaluated while assuming that they are preset with a low Reynolds number and a long wavelength. The analytical solution was deduced for temperature, concentration, axial velocity, tangential stress, and coefficient of heat transfer. Many emerging parameters and their effects on the aspects of the flow were illustrated, and the outcomes were expressed via graphs. Finally, some graphical presentations were made to assess the impacts of various parameters in a peristaltic motion of the fractional fluid in a tube of different nature. The present investigation is essential in many medical applications, such as the description of the gastric juice movement of the small intestine in inserting an endoscope.

\section{Introduction}

Numerous implementations have drawn interest of physicists, mathematicians, and engineers on magneto-hydrodynamic flow issues. In some applications and geothermal studies, metal alloy substantiation processes are optimized Sources, management of waste fuel, regulation of underground propagation and pollution of chemicals, waste, the construction of energy turbines for MHD, magnetic equipment for wound therapy and cancer tumour treatment, reduction of bleeding during surgery and transport of targeted magnetic particles as medicines. Several extensive works of literature on that fertile field are now available in $[1,2]$. Saqib et al. [3] clarified the nonlinear motion of the non-Newtonian fractional model fluid problem. Rashed and Ahmed [4] produced a numerical solution for dusty nanofluids peristaltic motion in a channel using a shooting method. The slip effect's problem on a peristaltic flow of the fractional fluid of second-grade over a cylindrical tube was examined by Rathod and Tuljappa [5]. Vajravelu et al. [6] obtained the velocity, temperature, and concentration with a magnetic field of a Carreau fluid in a channel with the heat and mass transfer. Ali et al. [7] discussed magnetic field effects on a blood flow that the blood was characterized as the Casson fluid. Zhao et al. [8] explored the motion natural convection temperature of a fraction with a magnetic field of viscoelastic fluid through a porous medium. Abd-Alla et al. [9] were researching the magnetic field's impact on a peristaltic motion of the fluid through the cylindrical cavity. Afzal et al. [10] analyzed the effect of the diffusivity convection and magnetic field in nanofluids on the peristaltic motion through the nonuniform channel. Heat and mass transfer's effects and magnetic field of the peristaltic motion in a planar channel were examined by Hayat and Hina [11]. The impact of the temperature and the magnetic field of peristaltic motion through a porous medium was debated by Srinivas and Kothandapani [12]. Ramzan et al. [13] discussed the heat flux and magnetic field's influences in Maxwell fluid flow through a two-way strained surface. Rachid [14] calculated the movement of viscoelastic fluid peristaltic transport under the Maxwell fractional model. The impact of a viscosity and a magnetic field of the peristaltic motion of synovial nanofluid in an asymmetric channel was reconnoitered by Ibrahim et al. [15]. Aly and Ebaid [16] inspected the slip conditions' effects of a peristaltic motion of nanofluids. Carrera et al. [17] checked the extension of a fractional 
Maxwell fluid and viscosity to the peristaltic motion. Zhao [18] exhibited the convection flow, the magnetic field, and velocity slip of a peristaltic motion of a fractional fluid. AbdAlla et al. [19] obtained the solution to the peristaltic motion problem in an endoscope tube. The analytical solution of the transport of viscoelastic fluid through a channel in the fractional peristalsis movement model was presented by Tripathi et al. [20]. The magnetic field effect on peristaltic movement in a vertical annulus was exposed by Nadeem and Akbar [21]. Srinivas et al. [22] were determining the effects on Newtonian fluid's peristaltic movement into porous channels of wall slip conditions, magnetic field, and heat transfer. Recent research expansions on the subject beginning from [23-33].

This paper aims to inspect the impacts of magnetic fields, heat and mass transfer, and fractional Maxwell fluids on the peristaltic flow of Jeffrey fluids. Both two-dimensional equations of motion and heat and mass transfer are generalized under the presence of low Reynolds numbers and a long wavelength. The temperature, concentration, axial velocity, tangential stress, and coefficient of heat transfer are empirical solutions, and the wave shape is found. In the problem, the relevant parameters are specified pictorially. The findings obtained are displayed and discussed graphically. For physicists, engineers, and individuals interested in developing fluid mechanics, the outcomes described in this paper are essential. The different potential fluid mechanical flow parameters for the Jeffrey peristaltic fluid are also supposed to serve as equally good theoretical estimates. Indeed, the current investigation is firmly believed to receive considerable attention from the researchers towards further peristaltic development with a variety of applications in physiological, modern technology, and engineering.

\section{Formulation of the Problem}

Take the MHD peristaltic flow through uniform coaxial tubes of a viscoelastic fluid through the fractional Maxwell fluid model. If the flow is transversely subject to a consistent magnetic field, electrical conductivity exists (Figure 1). Furthermore, it is supposed the inner and outer tube temperatures are $T_{0}$ and $T_{1}$, and concentrations are $C_{0}$ and $C_{1}$, respectively. We picked a cylindrical coordinate $\bar{R}$ and $\bar{Z}$. The equations for the tube walls are given by

$$
\begin{aligned}
& \overline{R_{1}}-a_{1}=0 \\
& \overline{R_{2}}-a_{2}=+b\left[\sin \frac{2 \pi}{\lambda}(\bar{Z}-c \bar{t})\right] .
\end{aligned}
$$

The equation of the fractional Maxwell fluid is given by

$$
\left(1+{\overline{\lambda_{1}}}^{\alpha_{1}} \widetilde{D}_{\bar{t}}^{\alpha_{1}}\right) \bar{S}=\mu \dot{\gamma},
$$

where $0 \leq \alpha_{1} \leq 1$.

$\widetilde{D}_{\bar{t}}^{\alpha_{1}}$ is defined as follows:

$$
\widetilde{D}_{\bar{t}}^{\alpha_{1}}(\bar{S})=D_{\bar{t}}^{\alpha_{1}}(\bar{S})+(\bar{V} \cdot \nabla)(\bar{S})-\bar{L}(\bar{S})-(\bar{S}) \bar{L}^{T},
$$

where

$$
\dot{\gamma}=(\nabla \bar{V})+(\nabla \bar{V})^{T}
$$

Also, note that $D_{\bar{t}}^{\alpha_{1}}=\partial_{\bar{t}}^{\alpha_{1}}$, of order $\alpha_{1}$ concerning $t$ and defined as follows:

$$
D_{\bar{t}}^{\alpha_{1}} f(t)=\frac{1}{\Gamma\left(1-\alpha_{1}\right)} \frac{\mathrm{d}}{\mathrm{d} t} \int_{0}^{t} \frac{f(\xi)}{(t-\xi)^{\alpha_{1}}} \mathrm{~d} \xi, \quad 0 \leq \alpha_{1} \leq 1
$$

The equation of motion can be written in the fixed frame which are derived $[32,33]$ as

$$
\begin{aligned}
& \rho\left[\frac{\partial}{\partial \bar{t}}+\bar{U} \frac{\partial}{\partial \bar{R}}+\bar{W} \frac{\partial}{\partial \bar{Z}}\right] \bar{U}+\frac{\partial \bar{p}}{\partial \bar{R}}=\frac{1}{\bar{R}} \frac{\partial}{\partial \bar{R}}\left(\bar{R}_{\overline{R R}}\right)+\frac{\partial}{\partial \bar{Z}}\left(\bar{S}_{\bar{R} Z}\right)-\frac{\bar{S}_{\overline{\theta \theta}}}{\bar{R}}, \\
& \rho\left[\frac{\partial}{\partial \bar{t}}+\bar{U} \frac{\partial}{\partial \bar{R}}+\bar{W} \frac{\partial}{\partial \bar{Z}}\right] \bar{W}+\frac{\partial \bar{p}}{\partial \bar{Z}}=\frac{1}{\bar{R}} \frac{\partial}{\partial \bar{R}}\left(\overline{R S}_{\bar{R} Z}\right)+\frac{\partial}{\partial \bar{Z}}\left(\bar{S}_{\overline{Z Z}}\right)+\rho g \alpha_{t}\left(\bar{T}-T_{o}\right) \\
& \rho g \alpha_{c}\left(\bar{C}-C_{o}\right)-\sigma B_{o}^{2} \bar{W} \\
& \rho_{C p}\left[\frac{\partial}{\partial \bar{t}}+\bar{U} \frac{\partial}{\partial \bar{R}}+\bar{W} \frac{\partial}{\partial \bar{Z}}\right] \bar{T}=K\left[\frac{\partial^{2}}{\partial \bar{R}^{2}}+\frac{1}{\bar{R}} \frac{\partial}{\partial \bar{R}}+\frac{\partial^{2}}{\partial \bar{Z}^{2}}\right] \bar{T}+Q_{o}, \\
& {\left[\frac{\partial}{\partial \bar{t}}+\bar{U} \frac{\partial}{\partial \bar{R}}+\bar{W} \frac{\partial}{\partial \bar{Z}}\right] \bar{C}=D_{m}\left[\frac{\partial^{2}}{\partial \bar{R}^{2}}+\frac{1}{\bar{R}} \frac{\partial}{\partial \bar{R}}+\frac{\partial^{2}}{\partial \bar{Z}^{2}}\right] \bar{C}+\frac{D_{m} K_{T}}{T_{m}}\left[\frac{\partial^{2}}{\partial \bar{R}^{2}}+\frac{1}{\bar{R}} \frac{\partial}{\partial \bar{R}}+\frac{\partial^{2}}{\partial \bar{Z}^{2}}\right] \bar{T},} \\
& \frac{\partial \bar{U}}{\partial \bar{R}}+\frac{\bar{U}}{\bar{R}}+\frac{\partial \bar{W}}{\partial \bar{Z}}=0 .
\end{aligned}
$$




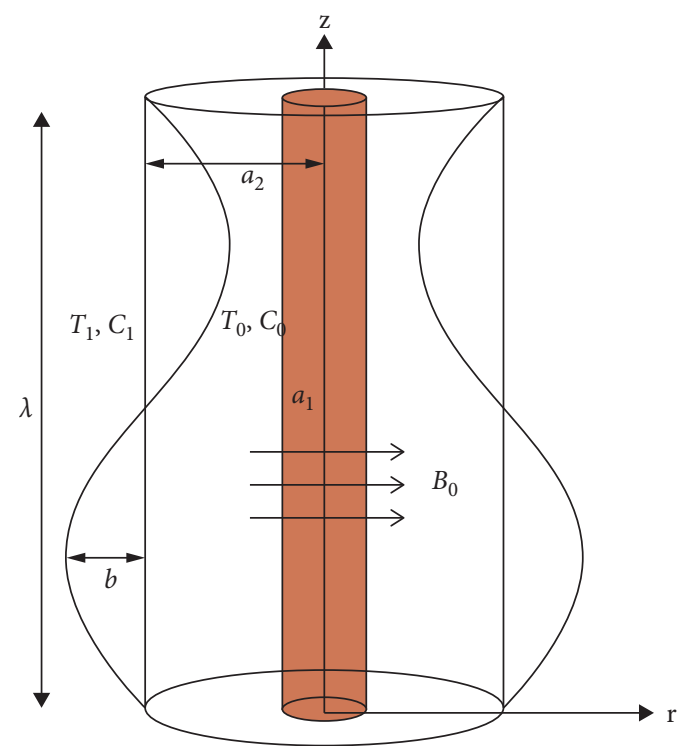

Figure 1: The geometry of the problem.

The transformation between these two frames can be written as follows:

$$
\begin{aligned}
& \bar{r}-\bar{R}=0, \\
& \bar{z}-\bar{Z}=-c \bar{t}, \\
& \bar{u}-\bar{U}=0, \\
& \bar{w}-\bar{W}=-c .
\end{aligned}
$$

The relevant governed boundary conditions for the considered flow analysis can be listed as

$$
\begin{gathered}
\bar{w}+c=0, \bar{u}=0 \text { at } \bar{r}=\overline{r_{1}}, \\
\bar{w}+c=0 \text { at } \bar{r}=\overline{r_{2}}+b \sin \left(\frac{2 \pi \bar{z}}{\lambda}\right), \\
\bar{T}-\overline{T_{1}}=0, \bar{C}-\overline{C_{1}}=0, \text { at } \bar{r}=\overline{r_{1}}, \\
\bar{T}-\overline{T_{0}}=0, \bar{C}-\overline{C_{0}}=0 \text { at } \bar{r}=\overline{r_{2}} .
\end{gathered}
$$

The leading motion equations of the flow for fluid in the wave frame are given by

$$
\begin{aligned}
& \rho\left[\bar{u} \frac{\partial}{\partial \bar{r}}+(\bar{w}+c) \frac{\partial}{\partial \bar{z}}\right] \bar{u}+\frac{\partial \bar{p}}{\partial \bar{r}}=\frac{1}{\bar{r}} \frac{\partial}{\partial r}\left(\bar{r} \bar{S}_{\overline{r r}}\right)+\frac{\partial}{\partial \bar{z}}\left(S_{\bar{r} \bar{z}}\right)-\frac{\bar{S}_{\overline{\theta \theta}}}{\bar{r}}, \\
& \rho\left[\bar{u} \frac{\partial}{\partial \bar{r}}+(\bar{w}+c) \frac{\partial}{\partial \bar{z}}\right] \bar{w}+\frac{\partial \bar{p}}{\partial \bar{z}}=\frac{1}{\bar{r}} \frac{\partial}{\partial \bar{r}}\left(\bar{r} \bar{S}_{\bar{r} \bar{z}}\right)+\frac{\partial}{\partial \bar{z}}\left(\bar{S}_{\overline{Z z}}\right)+\rho g \alpha_{t}\left(\bar{T}-T_{o}\right) \\
& \quad+\rho g \alpha_{c}\left(\bar{T}-T_{o}\right)-\sigma B_{o}^{2}(\bar{w}+c), \\
& \rho C_{p}\left[\bar{u} \frac{\partial}{\partial \bar{r}}+(\bar{w}+t c) \frac{\partial}{\partial \bar{z}}\right] \bar{T}=K\left[\frac{\partial^{2}}{\partial \bar{r}^{2}}+\frac{1}{r} \frac{\partial}{\partial \bar{r}}+\frac{\partial^{2}}{\partial \bar{z}^{2}}\right] \bar{T}+Q_{o}, \\
& {\left[\bar{u} \frac{\partial}{\partial \bar{r}}+(\bar{w}+c) \frac{\partial}{\partial \bar{z}}\right] \bar{C}=D_{m}\left[\frac{\partial^{2}}{\partial \bar{r}^{2}}+\frac{1}{r} \frac{\partial}{\partial \bar{r}}+\frac{\partial^{2}}{\partial \bar{z}^{2}}\right] \bar{C}+\frac{D_{m} K_{T}}{T_{m}}\left[\frac{\partial^{2}}{\partial \bar{r}^{2}}+\frac{1}{r} \frac{\partial}{\partial \bar{r}}+\frac{\partial^{2}}{\partial \bar{z}^{2}}\right] \bar{T},} \\
& \frac{\partial \bar{u}}{\partial \bar{r}}+\frac{\bar{u}}{\bar{r}}+\frac{\partial \bar{w}}{\partial \bar{z}}=0,
\end{aligned}
$$

where $\bar{S}$ depends only on $r$ and $t$. After using the initial condition $\bar{S}(\bar{t}=0)$, we find $\bar{S}_{\overline{r r}}=\bar{S}_{\overline{\theta \theta}}=\bar{S}_{\overline{z z}}=\bar{S}_{\bar{r} \bar{\theta}}=0$, and

$$
\left(1+\bar{\lambda}_{1}^{\alpha_{1}} \frac{\partial^{\alpha_{1}}}{\partial \bar{t}^{\alpha_{1}}}\right) \bar{S}_{\bar{r} \bar{z}}=\mu \frac{\partial \bar{w}}{\partial \bar{r}} .
$$


We present the following dimensionless parameters for further analysis:

$$
\begin{aligned}
& r=\frac{\bar{r}}{a_{2}}, \\
& z=\frac{\bar{z}}{\lambda} \text {, } \\
& t=\frac{c \bar{t}}{\lambda}, \\
& u=\frac{\bar{u}}{c \delta}, \\
& w=\frac{\bar{w}}{c}, \\
& \lambda_{1}=\frac{c \overline{\lambda_{1}}}{\lambda}, \\
& p=\frac{a_{2}^{2} \bar{p}}{c \lambda \mu}, \\
& \delta=\frac{a_{2}}{\lambda}, \\
& \theta=\frac{\bar{T}-T_{0}}{T_{1}-T_{0}}, \\
& \operatorname{Pr}=\frac{\mu C_{P}}{K} \text {, } \\
& \operatorname{Re}=\frac{\rho c a_{2}}{\mu}, \\
& \mathrm{Gr}=\frac{\rho g \alpha_{t}\left(T_{1}-T_{o}\right) a_{2}^{2}}{\mu c},
\end{aligned}
$$

$$
\begin{aligned}
M & =\sqrt{\frac{\sigma}{\mu}} B_{0} a_{2}, \\
S c & =\frac{\mu}{D_{m} \rho}, \\
\mathrm{Sr} & =\frac{\rho D_{m} K_{T}\left(T_{1}-T_{o}\right)}{\mu T_{m}\left(C_{1}-C_{0}\right)}, \\
\mathrm{Br} & =\frac{\rho g \alpha_{c}\left(C_{1}-C_{o}\right) a_{2}^{2}}{\mu c}, \\
\Theta & =\frac{\bar{C}-C_{0}}{C_{1}-C_{0}}, \\
\beta & =\frac{a_{2}^{2} Q_{o}}{K\left(T_{1}-T_{o}\right)}, \\
S & =\frac{a_{2} \bar{S}}{\mu c}, \\
r_{1} & =\frac{\frac{r_{1}}{a_{2}}}{r_{2}} \\
r_{2} & a_{2}
\end{aligned}
$$

wherever $\left(\varphi=\left(b / a_{2}\right)<1\right)$ is the wave amplitude.

\section{Solution of the Problem}

For the abovementioned modifications and nondimensional variables listed earlier, the preceding equations are reduced to

$$
\begin{aligned}
& \operatorname{Re} \delta^{3}\left[u \frac{\partial}{\partial r}+(w+1) \frac{\partial}{\partial z}\right] u+\frac{\partial p}{\partial r}=\frac{\delta}{r} \frac{\partial}{\partial r}\left(r S_{r r}\right)+\delta^{2} \frac{\partial}{\partial z}\left(S_{r z}\right)-\delta\left(\frac{S_{\theta \theta}}{r}\right) \\
& \operatorname{Re} \delta\left[u \frac{\partial}{\partial r}+(w+1) \frac{\partial}{\partial z}\right] w+\frac{\partial p}{\partial z}=\frac{1}{r} \frac{\partial}{\partial r}\left(r S_{r z}\right)+\delta \frac{\partial}{\partial z}\left(S_{z z}\right)+\operatorname{Gr} \theta+\operatorname{Br} \Theta-M^{2}(w+1) \\
& \operatorname{RePr} \delta\left[u \frac{\partial}{\partial r}+(w+1) \frac{\partial}{\partial z}\right] \theta=\left[\frac{\partial^{2}}{\partial r^{2}}+\frac{1}{r} \frac{\partial}{\partial r}+\delta^{2} \frac{\partial^{2}}{\partial z^{2}}\right] \theta+\beta \\
& \operatorname{Re} \delta\left[u \frac{\partial}{\partial r}+(w+1) \frac{\partial}{\partial z}\right] \Theta=\frac{1}{\operatorname{Sc}}\left[\frac{\partial^{2}}{\partial r^{2}}+\frac{1}{r} \frac{\partial}{\partial r}+\delta^{2} \frac{\partial^{2}}{\partial z^{2}}\right] \Theta+\operatorname{Sr}\left[\frac{\partial^{2}}{\partial r^{2}}+\frac{1}{r} \frac{\partial}{\partial r}+\delta^{2} \frac{\partial^{2}}{\partial z^{2}}\right] \theta \\
& \frac{\partial u}{\partial r}+\frac{u}{r}+\frac{\partial w}{\partial z}=0 .
\end{aligned}
$$

With boundary conditions

$$
\begin{aligned}
w+1 & =0, u=0 \text { at } r=r_{1}=\varepsilon, \\
w+1 & =0 \text { at } r=r_{2}=1+\varphi \sin (2 \pi z), \\
\theta-1 & =0, \Theta-1=0 \text { at } r=r_{1}, \\
\theta & =0, \Theta=0 \text { at } r=r_{2} .
\end{aligned}
$$

\section{The Analytical Solution}

Furthermore, the hypothesis of the long wavelength approach is also supposed. Now, $\delta$ is very small so that it can be tended to zero. Thus, the $\delta \ll 1$ dimensionless governing equations (12)-(15) by using this hypothesis may be written as 


$$
\begin{aligned}
\frac{\partial p}{\partial r} & =0 \\
f\left[\frac{\mathrm{d} p}{\mathrm{~d} z}-\mathrm{Gr} \theta-\operatorname{Br} \Theta+M^{2}(w+1)\right] & =\left(\frac{\partial^{2} w}{\partial r^{2}}+\frac{1}{r} \frac{\partial w}{\partial r}\right) \\
\frac{\partial^{2} \theta}{\partial r^{2}}+\frac{1}{r} \frac{\partial \theta}{\partial r}+\beta & =0 \\
\frac{1}{\mathrm{Sc}}\left[\frac{1}{r} \frac{\mathrm{d}}{\mathrm{d} r}\left(r \frac{\partial \Theta}{\partial r}\right)\right]+\operatorname{Sr}\left[\frac{1}{r} \frac{\mathrm{d}}{\mathrm{d} r}\left(r \frac{\partial \theta}{\partial r}\right)\right] & =0
\end{aligned}
$$

equation (18) specifies that $p$ is only a function of $z$.

Temperature, concentration, and axial velocity solutions can be described as follows:

$\theta=\frac{\log \left(r / r_{2}\right)}{\log \left(r_{1} / r_{2}\right)}+\frac{\beta}{4}\left[\frac{r_{1}^{2} \log \left(r / r_{2}\right)-r_{2}^{2} \log \left(r / r_{1}\right)}{\log \left(r_{1} / r_{2}\right)}\right]-\frac{\beta}{4} r^{2}$,

$\Theta=\frac{\operatorname{SrSc} \beta}{4} r^{2}+c_{1} \log (r)+c_{2}$,

$w=4 c_{2}+4 c_{1} \log (r)+f \times \frac{\left[\begin{array}{c}(\mathrm{d} p / \mathrm{d} z) r^{2}-\operatorname{Gr}\left(\left(r^{2}\left(\log \left(r / r_{2}\right)-1\right) / \log \left(r_{1} / r_{2}\right)\right)+(\beta / 4)\left(r_{1}^{2} r^{2}\left(\log \left(r / r_{2}\right)-1\right)-r_{2}^{2} r^{2}\left(\log \left(r / r_{1}\right)-1\right) / \log \left(r_{1} / r_{2}\right)\right)-(\beta / 16) r^{4}\right) \\ -\operatorname{Br}\left(\left(\operatorname{SrSc} \beta \times r^{4} / 16\right)+c_{1} r^{2}(\log (r)-1)+c_{2} r^{2}\right)+M^{2} r^{2}\end{array}\right]}{\left[4-f M^{2} r^{2}\right]}$,

where

$$
\begin{aligned}
f & =\left(1+\lambda_{1}^{\alpha_{1}} D_{t}^{\alpha_{1}}\right), \\
A & =-4-f \times\left[\frac{\mathrm{d} p}{\mathrm{~d} z} r_{1}^{2}+\operatorname{Gr}\left(\frac{r_{1}^{2}\left(\log \left(r_{1} / r_{2}\right)-1\right)}{\log \left(r_{1} / r_{2}\right)}+\frac{\beta}{4}\left(\frac{r_{1}^{4}\left(\log \left(r_{1} / r_{2}\right)-1\right)+r_{1}^{2} r_{2}^{2}}{\log \left(r_{1} / r_{2}\right)}\right)-\frac{\beta}{16} r_{1}^{4}\right)\right], \\
B & =-4-f \times\left[\frac{\mathrm{d} p}{\mathrm{~d} z} r_{2}^{2}+\operatorname{Gr}\left(\frac{-r_{2}^{2}}{\log \left(r_{1} / r_{2}\right)}+\frac{\beta}{4}\left(\frac{-r_{1}^{2} r_{2}^{2}-r_{2}^{4}\left(\log \left(r_{1} / r_{2}\right)-1\right)}{\log \left(r_{1} / r_{2}\right)}\right)-\frac{\beta}{16} r_{2}^{4}\right)\right], \\
C_{1} & =\frac{4+\operatorname{SrSc} \beta\left(r_{2}^{2}-r_{1}^{2}\right)}{4 \log \left(r_{1} / r_{2}\right)}, \\
C_{2} & =\frac{\operatorname{SrSc} \beta\left[r_{1}^{2} \log \left(r_{2}\right)-r_{2}^{2} \log \left(r_{1}\right)\right]-4 \log \left(r_{2}\right)}{4 \log \left(r_{1} / r_{2}\right)}, \\
C_{3} & =\frac{(A-B)}{4 \log \left(r_{1} / r_{2}\right)}, \\
C_{4} & =\frac{\left(B \log \left(r_{1}\right)-A \log \left(r_{2}\right)\right)}{4 \log \left(r_{1} / r_{2}\right)} .
\end{aligned}
$$

The heat transfer coefficient is indicated as follows:

So, the solution of heat transfer is given by

$$
Z r=\frac{\partial \theta}{\partial r} \times \frac{\partial r_{2}}{\partial z}
$$

$$
Z r=\left[-\frac{r \beta}{2}+\frac{1}{r \log \left(r_{1} / r_{2}\right)}+\frac{\left(\left(r_{1}^{2} / r\right)-\left(r_{2}^{2} / r\right)\right) \beta}{4 \log \left(r_{1} / r_{2}\right)}\right] \times[2 \varphi \pi \cos (2 \pi z)]
$$




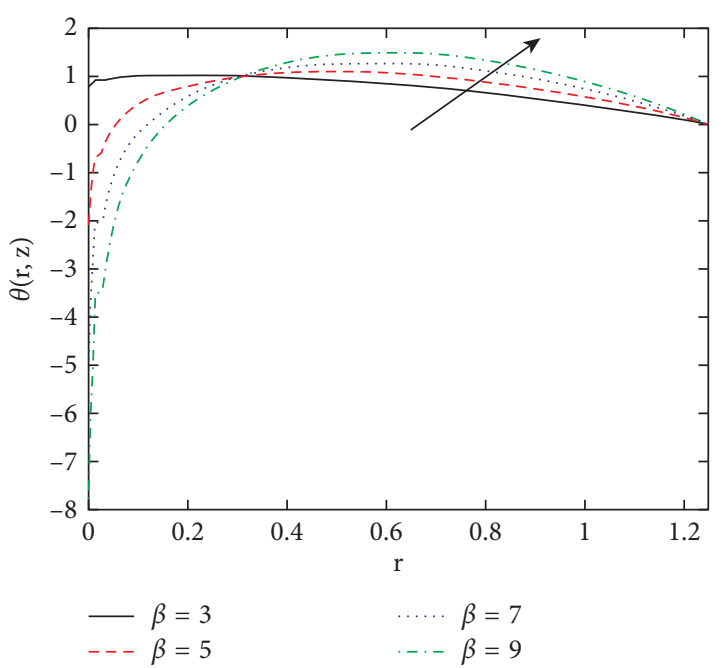

(a)

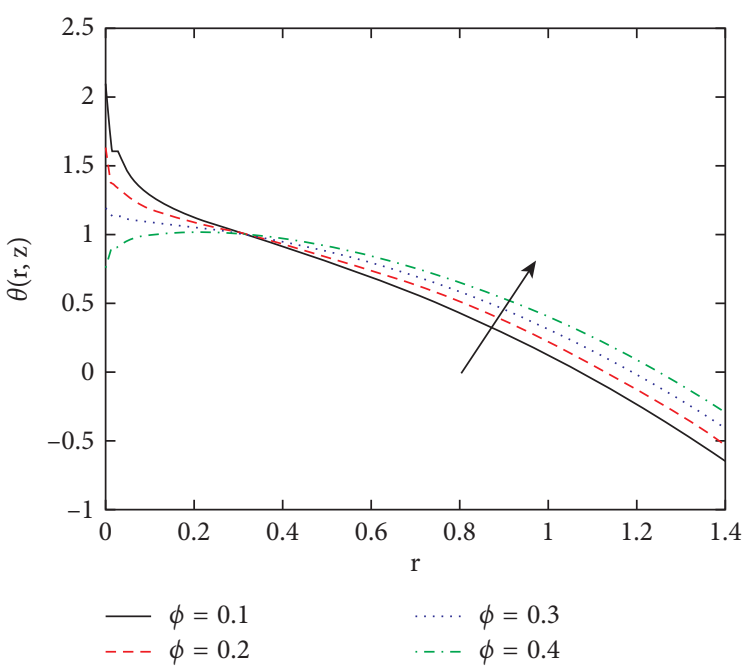

(b)

FIgURE 2: Discrepancies of the temperature $\theta$ against the $r$-axis for various values of $\beta$ and $\varphi$..

Using the definition of the fractional differential operator (5) we find the expression of $f$ as follows:

$$
f=f(t)=1+\lambda_{1}^{\alpha_{1}} \frac{t^{-\alpha_{1}}}{\Gamma\left(1-\alpha_{1}\right)}
$$

\section{Results and Discussion}

In this section, the effect of different parameters is shown graphically in Figures 2-7 such as fractional parameter $\alpha_{1}$, heat source/sink parameter $\beta$, wave amplitude $\varphi$, radius ratio $\varepsilon$, Hatman number $M$, Grashof number $\mathrm{Gr}$, relaxation time $\lambda_{1}$, the Soret number Sr, and the Schmidt number Sc on the temperature $\theta$, the concentration $\Theta$, axial velocity $w$, tangential stress $s_{r z}$, and heat transfer coefficient $Z r$. MATLAB software is used to identify the quantitative influences of various physical parameters implicated in the our study. Approximate analytical results are numerically evaluated for temperature, concentration, axial velocity, tangential stress, and the heat transfer coefficient for various values of parameters. For this object, Figures 2-7 are displayed.

Figure 2 has been plotted to clarify the variations of $\beta$ and $\varphi$ on the temperature distribution $\theta$. Figure 2 shows that $\theta$ decreases when $\beta$ increases in the range $0 \leq r \leq 0.32$, while $\theta$ increases when $\beta$ increases in the range $0.32 \leq r \leq 1.2$. Moreover, $\theta$ decreases when $\varphi$ increases in the range $0 \leq r \leq 0.32$, while $\theta$ increases when $\varphi$ increases in the range $0.32 \leq r \leq 1.4$. In addition, the temperature decreases with the radial increase and the boundary conditions are fulfilled.

Figure 3 displays the discrepancy of the concentration with the radial for various values of $\varepsilon, \varphi$, Sc and Sr. It is indicated that the concentration increases with increasing $\varepsilon$ and $\varphi$. However, $\Theta$ decreases with increasing Sr and Sc. In addition, the concentration decreases with the radial increase and the boundary conditions are fulfilled.
The impacts of $\mathrm{Gr}, \lambda_{1}, \varphi, \alpha_{1}, M$, and $S c$ on the axial velocity $w$ are illustrated in Figure 4. It is indicated that the axial velocity profiles decreases with increasing $\mathrm{Gr}, \lambda_{1}$, and $\varphi$ in the range $0 \leq r \leq 0.32$, while it increases in the range $0.32 \leq r \leq 0.45$, In addition to this, the axial velocity profile decreases with increasing $\alpha_{1}$ in the whole range $0 \leq z \leq 1$, while it increases with increasing $M$ in the whole range $0 \leq z \leq 1$, the axial velocity profiles decreases with increasing Sc in the range $0 \leq z \leq 53$ as well, and it increases in the range $0.53 \leq r \leq 0.88$ and then decreases again in the range $0.88 \leq z \leq 1$. Also, it is observed that the velocity has oscillatory behavior due to peristaltic motion concerned.

The effect of $\alpha_{1}, M, \beta$ and Sc can be observed from Figure 5, in which the tangential stress is illustrated for the various values of $\alpha_{1}, M, \beta$, and Sc. With the increase of $\alpha_{1}$ and Sc, the tangential stress decreases. Moreover, tangential stress increases with increasing $M$ and $\beta$. It is noticed that one can observe the tangential stress is in oscillatory behavior, which may be due to peristalsis.

Figure 6 explains the influence of $\varepsilon$ and $\varphi$ on the heat transfer coefficient $Z h$. Obviously, the increase in $\varepsilon$ and $\varphi$ increases the amplitude of the heat transfer coefficient in the whole range $z$. From Figure 6, one can observe that heat transfer coefficient is an oscillatory behavior in the whole range, which may be due to peristalsis.

Figure 7 is plotted in $3 \mathrm{D}$ schematics concern the axial velocity $w$, the concentration $\Theta$, the temperature $\theta$, and the heat transfer coefficient $Z h$ concerning $r$ and $z$ axes in the presence $\alpha_{1}, \mathrm{Sr}, \varepsilon$, and $\varphi$. It is indicated that the axial velocity decreases by increasing $\alpha_{1}$, Also, the concentration decreases by increasing $\mathrm{Sr}$, the temperature increases with increasing of $\varepsilon$ as well, otherwise the heat transfer coefficient increases by increasing $\varphi$. For all physical quantities, we obtain the peristaltic flow in $3 \mathrm{D}$ overlapping and damping when the state of particle equilibrium is reached and increased. The vertical distance of the curves is greater, with most physical fields moving in peristaltic flow. 

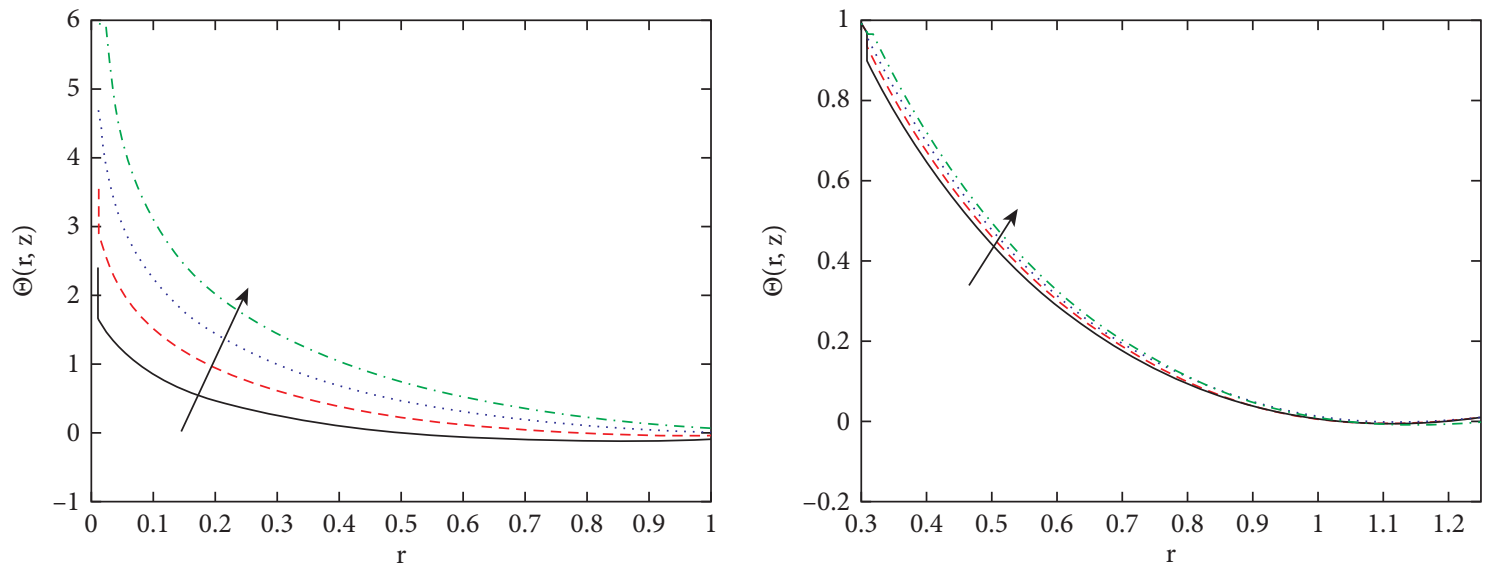

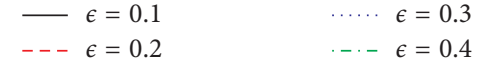

(a)

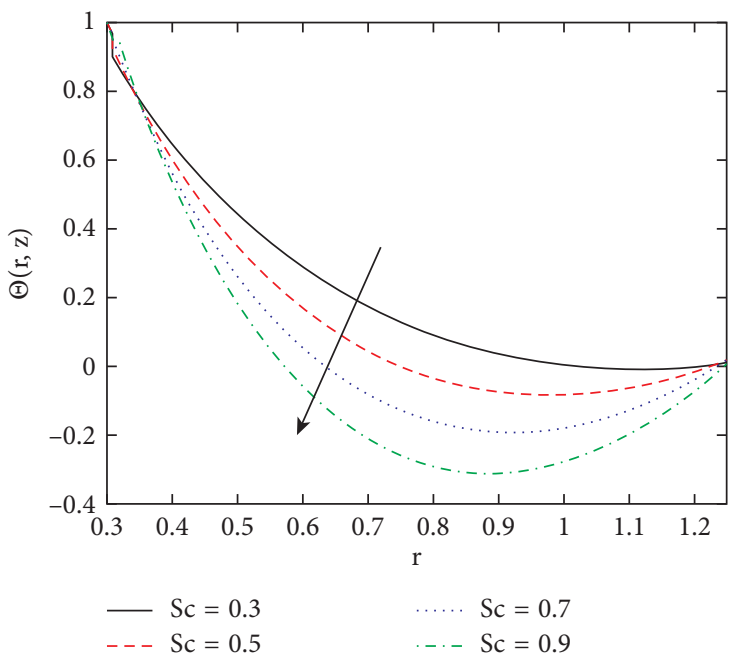

(c)

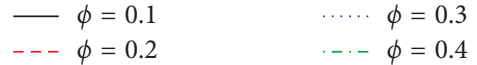

(b)

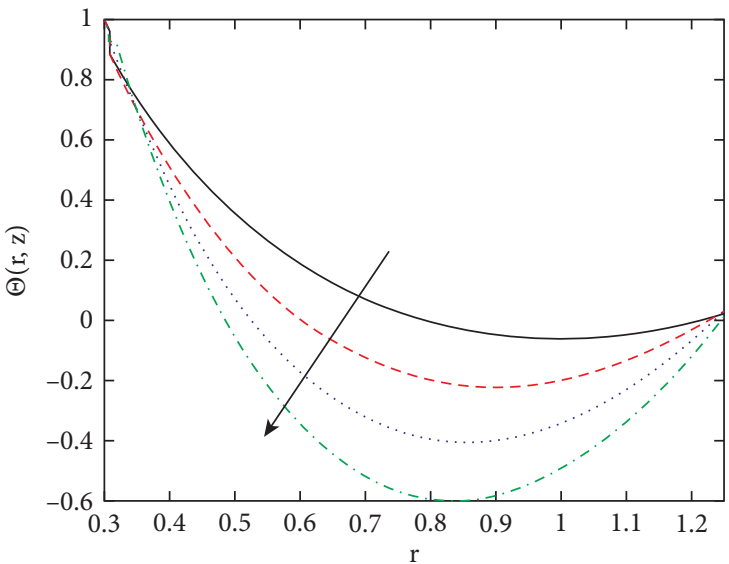

Sr $=3$

-- Sr $=5$

(d)

Figure 3: Discrepancies of the concentration $\Theta$ against the $r$-axis for various values of $\varepsilon, \varphi$, Sc, and Sr.
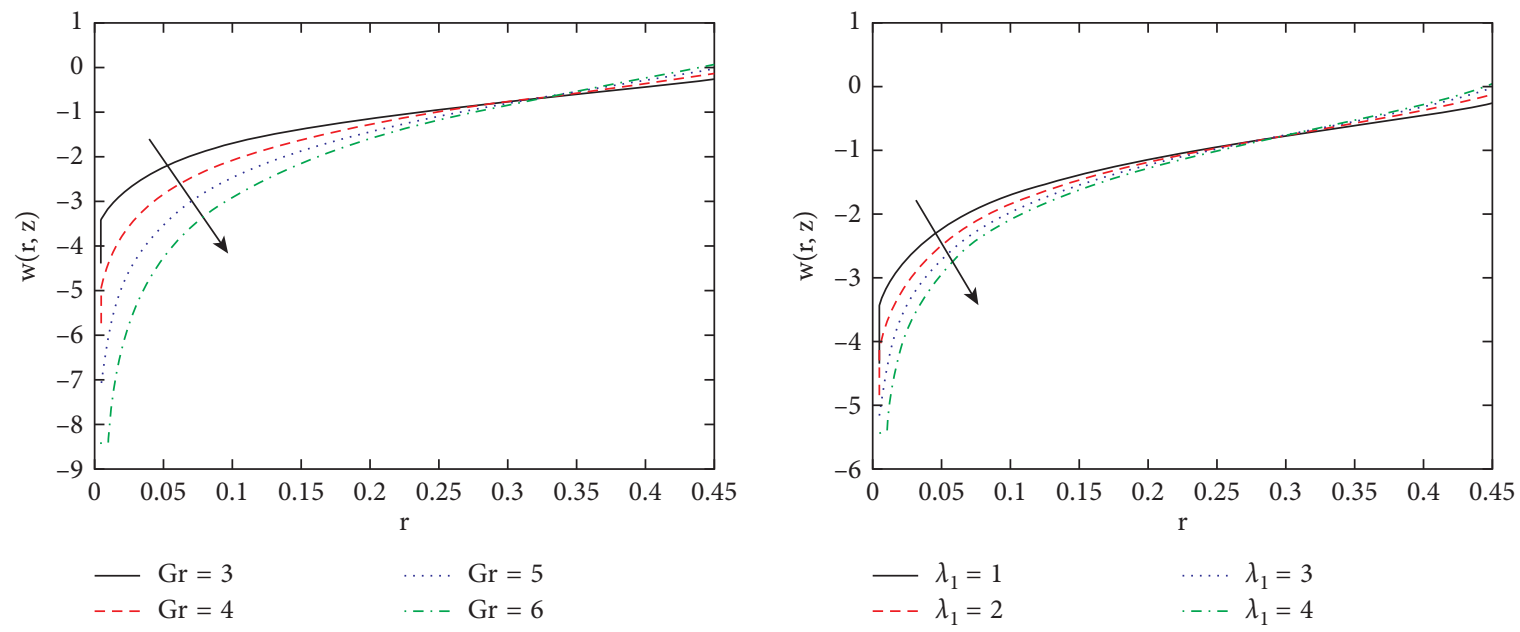

(a)

(b)

FIgUre 4: Continued. 

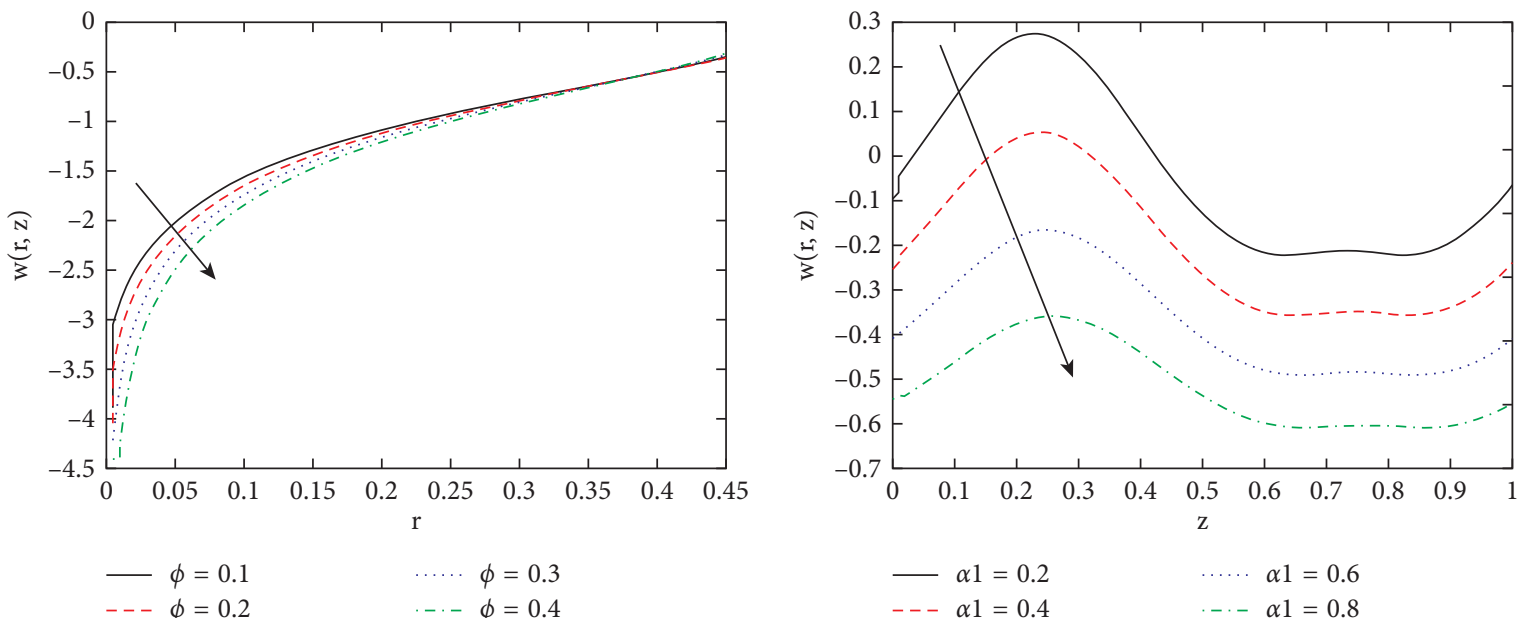

(c)

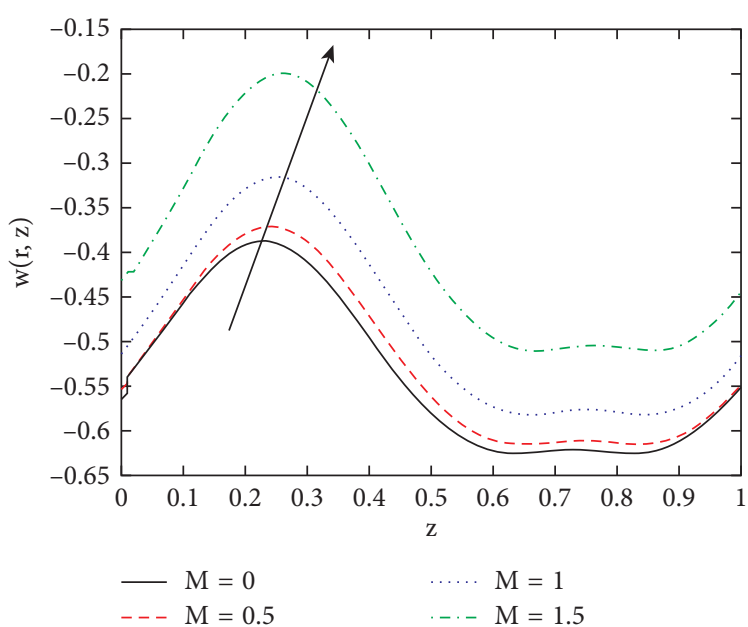

(e)

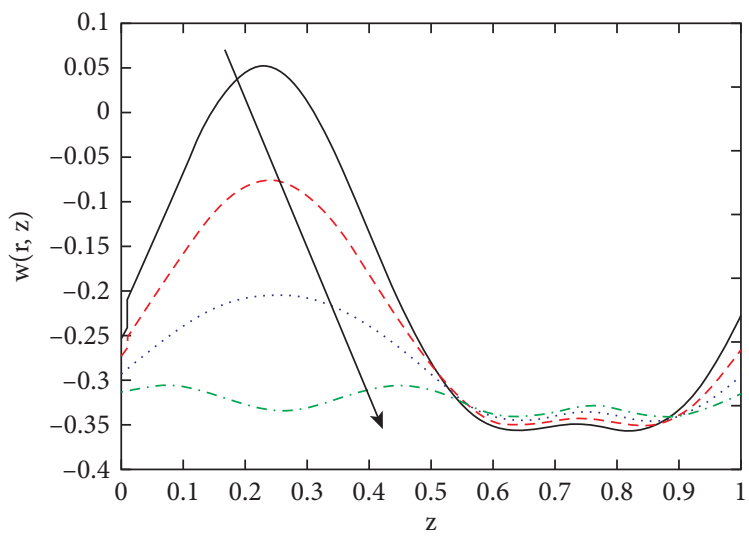

$\begin{aligned}-\mathrm{Sc}=0.3 & \ldots . \mathrm{Sc}=0.7 \\ --\mathrm{Sc}=0.5 & -.-\mathrm{Sc}=0.9\end{aligned}$

(f)

Figure 4: Discrepancies of the axial velocity $w$ against the $r$ - and the $z$-axes for various values of $\lambda_{1} \lambda_{1}, \varphi, \alpha_{1}, M$, and Sc.
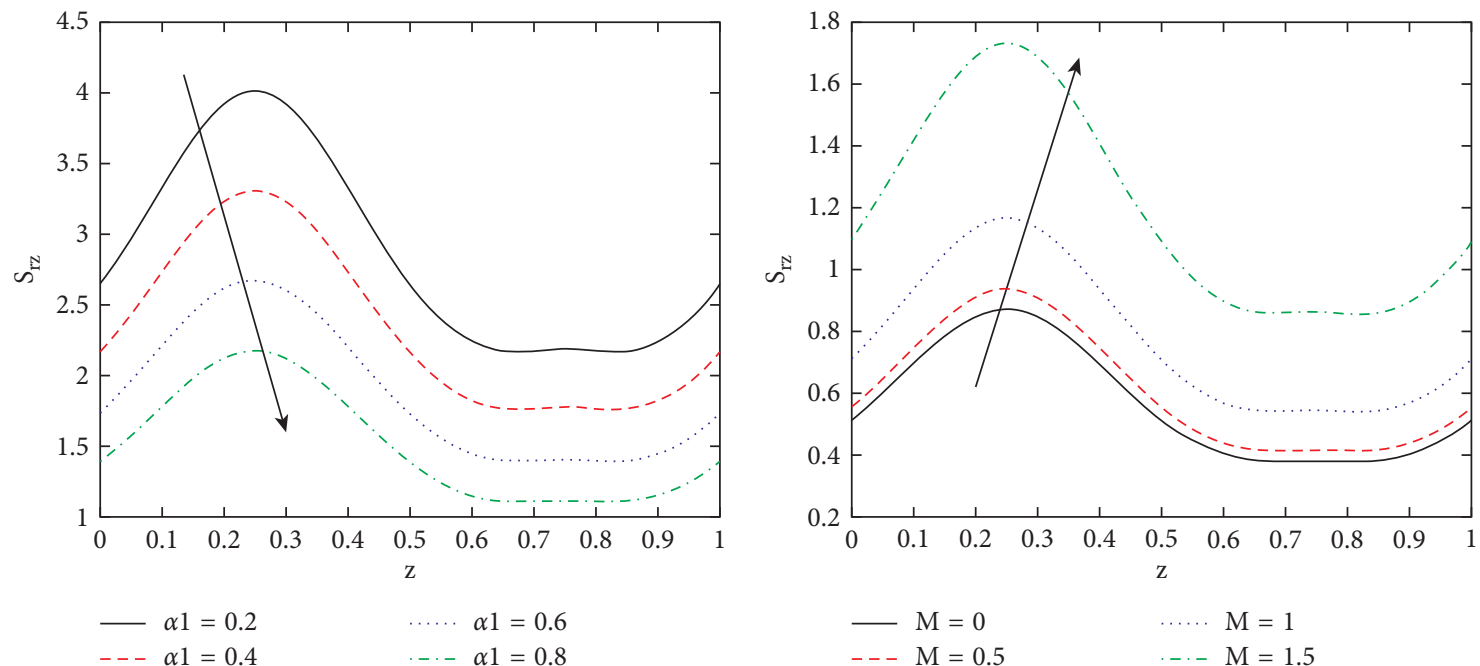

$\begin{aligned} \alpha 1 & =0.2 \\ --\alpha 1 & =0.4\end{aligned}$

(a)

(b)

Figure 5: Continued. 

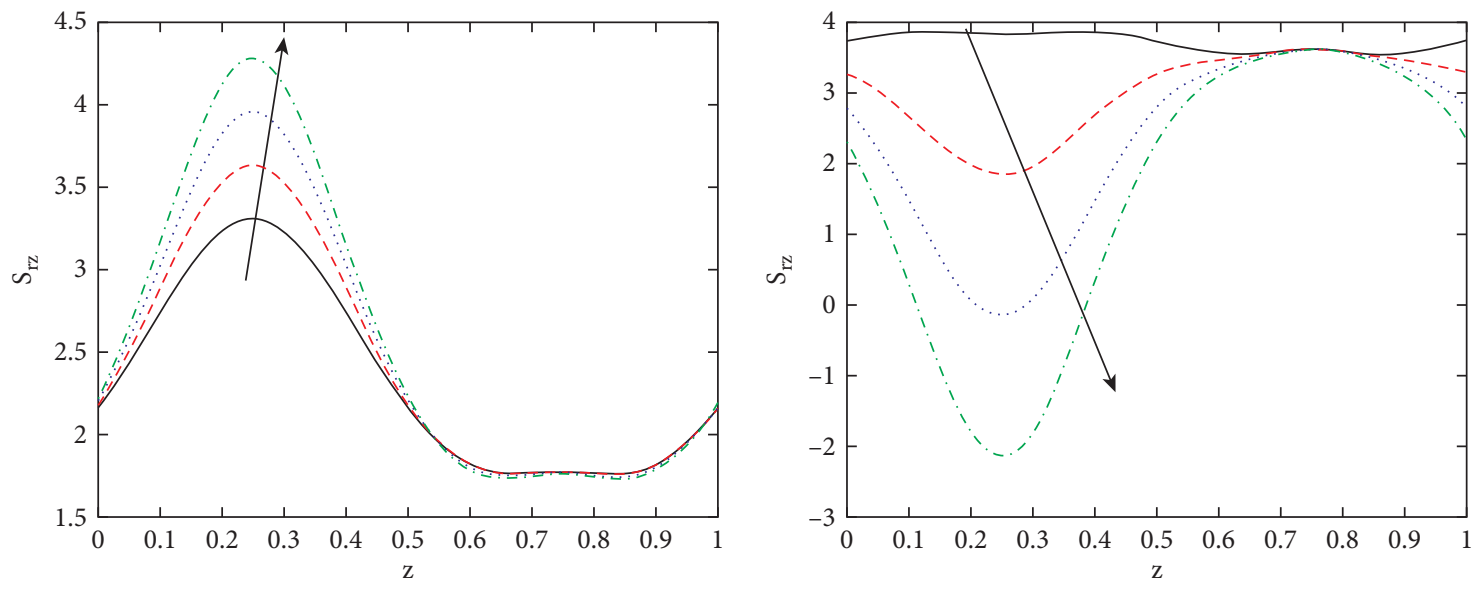

$\begin{array}{ll}-\beta=3 & \cdots \cdots \\ --\beta=5 & \cdots-\beta=9\end{array}$

(c)
$-\mathrm{Sc}=0.3$
- - - Sc $=0.5$
-.. Sc $=0.9$

(d)

FIGURE 5: Discrepancies of the axial tangential stress $s_{r z}$ against the $z$-axis for various values of $\alpha_{1}, M, \beta$, and Sc.

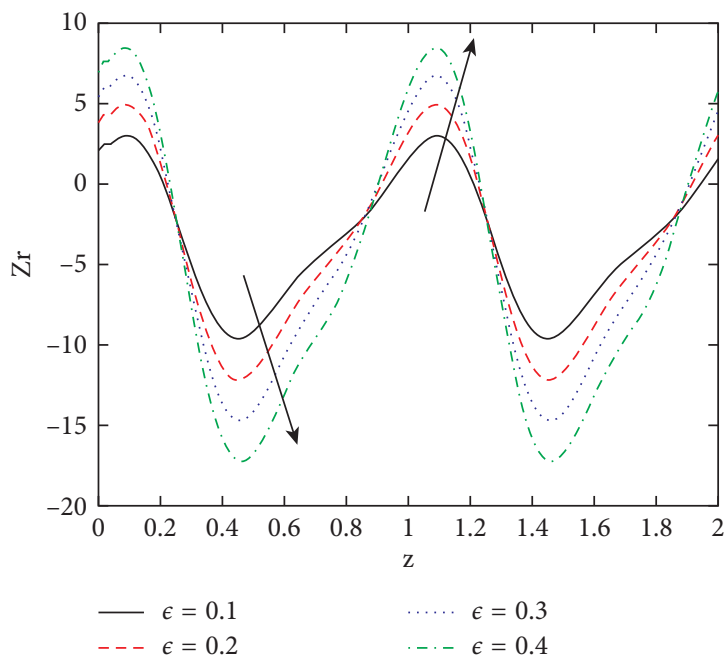

(a)

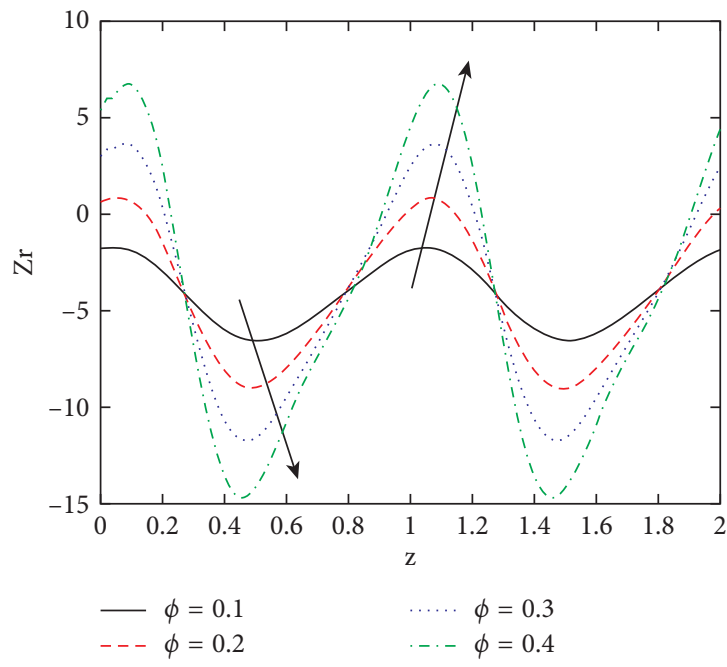

(b)

Figure 6: Discrepancies of the heat transfer coefficient $Z r$ against the $z$-axis for various values of $\varepsilon$ and $\varphi$.

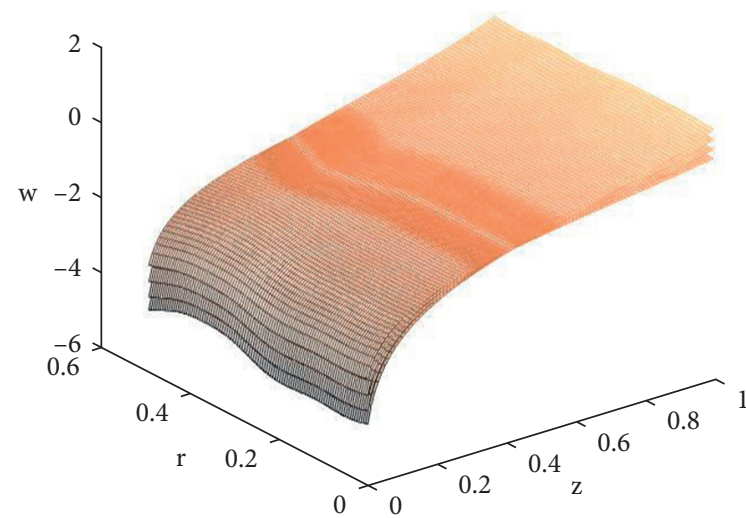

(a)

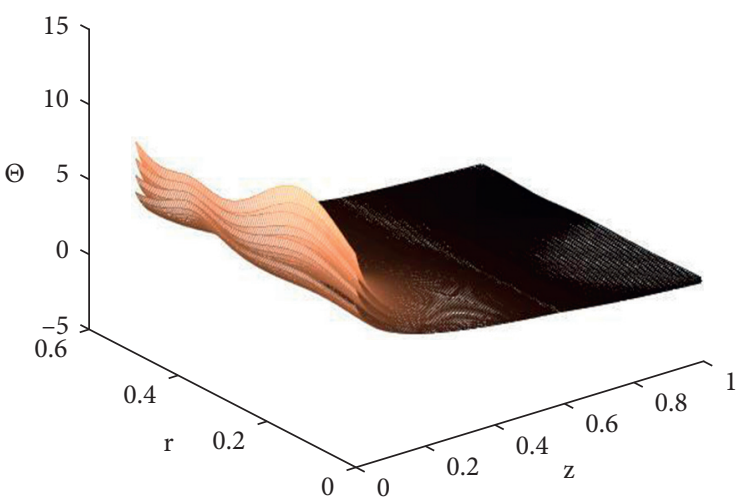

(b)

Figure 7: Continued. 


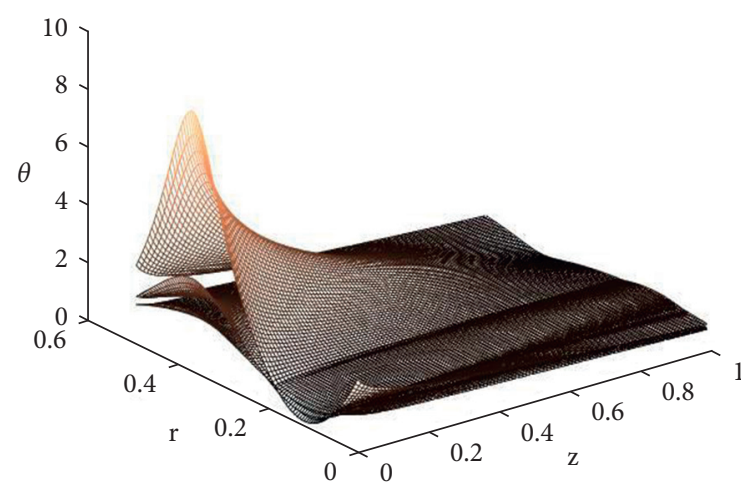

(c)

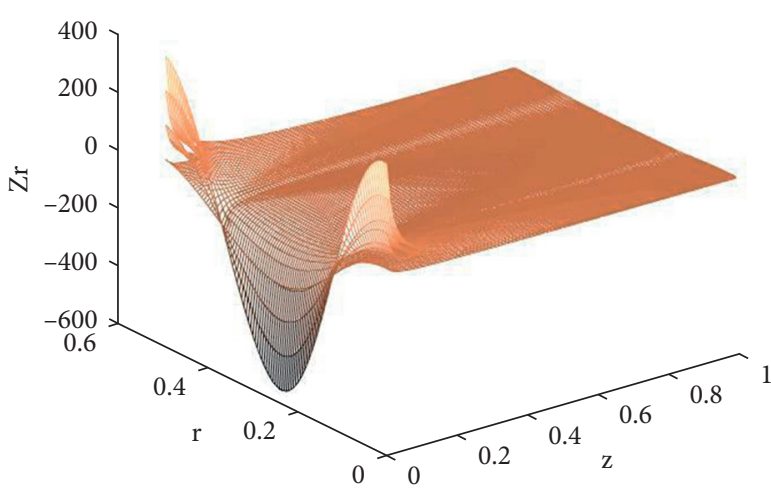

(d)

Figure 7: Discrepancies of the axial velocity, $w$, the concentration, $\Theta$, the temperature, $\theta$, and the heat transfer coefficient $Z r$ in $3 \mathrm{D}$ against $r$ - and $z$-axis under the influence of $\alpha_{1}, \operatorname{Sr}, \varepsilon$, and $\varphi$..

\section{Conclusions}

The concluding remarks are listed as follows:

(1) The axial velocity decreases and increases with the increase of $\alpha_{1}, \varphi, \mathrm{Gr}, \lambda_{1}$, and Sc due to the increase in the Lorentz force.

(1) The temperature increases with the increase of the wave amplitude and radius ratio.

(2) The concentration decreases with the increase of both $\mathrm{Sr}, \mathrm{Sc}$ and it increases with the increase of both $\varepsilon$ and $\varphi$.

(3) The tangential stress decreases and increases with the increase of both $\alpha_{1}$, Sc, and it increases with the increase both $M$ and $\beta$.

(4) The study of the phenomenon under effect of $\alpha_{1}, \beta, \varphi$, $\varepsilon, M, \mathrm{Gr}, \lambda_{1}, \mathrm{Sr}$, and $\mathrm{Sc}$ was performed.

(5) This study has indeed been widely applied in many fields of science, such as medicine and the medical industry. Thus, in the field of fluid mechanics, it is considered as extremely essential. When inserting an endoscope through the small intestine, this study describes the movement of the gastric juice.

\section{Nomenclature}

$\overline{R_{1}}, \overline{R_{2}}$ : Shapes of the wave walls

$t: \quad$ Time in a wave frame

$\overline{\lambda_{1}}: \quad$ Relaxation time

$\alpha_{1}$ : $\quad$ Fractional time derivative parameter

$\dot{\gamma}: \quad$ Rate of the shear strain

$\bar{U}, \bar{W}$ : The components of the velocity in a laboratory frame

$\bar{u}, \bar{w}: \quad$ The components of the velocity in a wave frame

$\bar{P}$ : $\quad$ The pressure in a laboratory frame

$\bar{p}: \quad$ The pressure in a wave frame

$\sigma: \quad$ Fluid's electric conductance

$B_{o}$ : The intensity of the external magnetic field

$\rho: \quad$ Density

$g: \quad$ Gravity constant

$\alpha_{t}$ : Linear coefficient of the thermal expansion $\alpha_{c}$ : Coefficient of the viscosity at constant concentration

$c_{p}: \quad$ Specific heat

K: $\quad$ Thermal conductivity

$Q_{O}: \quad$ Heat generation coefficient

$\varphi$ : Wave amplitude in the dimensionless form

$\varepsilon: \quad$ Radius ratio

$\theta$ : The distribution of temperature

$\Theta: \quad$ The distribution of concentration

$T_{0}, T_{1}$ : Inner and outer tube temperature

$C_{0}, C_{1}$ : Inner and outer tube concentration

$\delta$ : Wavenumber

$\mu: \quad$ Fluid viscosity

M: Hartmann number

Re: $\quad$ Reynolds number

Pr: $\quad$ Prandtl number

Gr: $\quad$ Grashof number

$\beta$ : $\quad$ The heat source/sink parameter

Br: Brinkman number

Sr: $\quad$ Soret number

Sc: $\quad$ Schmidt number.

\section{Data Availability}

No data were used to support this study.

\section{Conflicts of Interest}

The authors declare that they have no conflicts of interest.

\section{Acknowledgments}

The authors would like to express their gratitude to Taif University for supporting the present study under Taif University Researchers Supporting Project numbered (TURSP-2020/164).

\section{References}

[1] A. M. Abd-Alla, S. M. Abo-Dahab, and H. D. El-Shahrany, "Effects of rotation and initial stress on peristaltic transport of fourth grade fluid with heat transfer and induced magnetic 
field," Journal of Magnetism and Magnetic Materials, vol. 349, pp. 268-280, 2014.

[2] A. M. Abd-Alla, G. A. Yahya, S. R. Mahmoud, and H. S. Alosaimi, "Effect of the rotation, magnetic field and initial stress on peristaltic motion of micropolar fluid," Meccanica, vol. 47, pp. 1455-1465, 2012.

[3] M. Saqib, H. Hanif, T. Abdeljawad, I. Khan, S. Shafie, and K. S. Nisar, "Heat transfer in MHD flow of Maxwell fluid via fractional Cattaneo-Friedrich model," Computers, Materials \& Continua, vol. 65, no. 3, pp. 1959-1973, 2020.

[4] Z. Z. Rashed and S. E. Ahmed, "Peristaltic flow of dusty nanofluids in curved channels," Computers, Materials \& Continua, vol. 66, no. 1, pp. 1012-1026, 2021.

[5] V. P. Rathod and A. Tuljappa, "Slip effect on the peristaltic flow of a fractional second grade fluid through a cylindrical tube," Advances in Applied Science Research, vol. 6, no. 3, pp. 101-111, 2015.

[6] K. Vajravelu, S. Sreenadh, and R. Saravana, "Combined influence of velocity slip, temperature and concentration jump conditions on MHD peristaltic transport of a Carreau fluid in a non-uniform channel," Applied Mathematics and Computation, vol. 225, pp. 656-676, 2013.

[7] F. Ali, N. A. Sheikh, I. Khan, and M. Saqib, "Magnetic field effect on blood flow of Casson fluid in axisymmetric cylindrical tube: a fractional model," Journal of Magnetism and Magnetic Materials, vol. 423, no. 4, pp. 327-336, 2017.

[8] J. Zhao, L. Zheng, X. Zhang, and F. Liu, "Convection heat and mass transfer of fractional MHD Maxwell fluid in a porous medium with Soret and Dufour effects," International Journal of Heat and Mass Transfer, vol. 103, pp. 203-210, 2016.

[9] A. M. Abd-Alla, S. M. Abo-Dahab, and A. Kilicman, "Peristaltic flow of a Jeffrey fluid under the effect of radially varying magnetic field in a tube with an endoscope," Journal of Magnetism and Magnetic Materials, vol. 384, no. 15, pp. 79-86, 2015.

[10] Q. Afzal, S. Akram, R. Ellahi, S. M. Sait, and F. Chaudhry, "Thermal and concentration convection in nanofluids for peristaltic flow of magneto couple stress fluid in a non uniform channel," Journal of Thermal Analysis and Calorimetry, vol. 143, no. 2, pp. 56-87, 2021.

[11] T. Hayat and S. Hina, "The influence of wall properties on the MHD peristaltic flow of a Maxwell fluid with heat and mass transfer," Nonlinear Analysis: Real World Applications, vol. 11, no. 4, pp. 3155-3169, 2010.

[12] S. Srinivas and M. Kothandapani, "the influence of heat and mass transfer on MHD peristaltic flow through a porous space with compliant walls," Applied Mathematics and Computation, vol. 213, no. 1, pp. 197-208, 2009.

[13] M. Ramzan, M. Bilal, and J. D. Chung, "Influence of homogeneous-heterogeneous reactions on MHD 3D Maxwell fluid flow with Cattaneo-Christov heat flux and convective boundary condition," Journal of Molecular Liquids, vol. 230, pp. 415-422, 2017.

[14] H. Rachid, "Effects of heat transfer and an endoscope on peristaltic flow of a fractional maxwell fluid in a vertical tube," Abstract and Applied Analysis, vol. 2015, Article ID 360918, 9 pages, 2015.

[15] M. G. Ibrahim, W. M. Hasona, and A. A. ElShekhipy, "Concentration-dependent viscosity and thermal radiation effects on MHD peristaltic motion of Synovial nanofluid: applications to rheumatoid arthritis treatment," Computer Methods and Programs in Biomedicine, vol. 170, pp. 39-52, 2019.

[16] E. H. Aly and A. Ebaid, "Exact analytical solution for the peristaltic flow of nanofluids in an asymmetric channel with slip effect of the velocity, temperature and concentration," Journal of Mechanics, vol. 30, no. 4, pp. 411-422, 2014.

[17] Y. Carrera, G. A. Rosa, E. J. Vernon-Carter, and J. AlvarezRamirez, "A fractional-order Maxwell model for nonNewtonian fluids," Physica A: Statistical Mechanics and Its Applications, vol. 482, pp. 276-285, 2017.

[18] J. Zhao, "Axisymmetric convection flow of fractional Maxwell fluid past a vertical cylinder with velocity slip and temperature jump," Chinese Journal of Physics, vol. 67, pp. 501-511, 2020.

[19] A. M. Abd-Alla, S. M. Abo-Dahab, and R. D. El-Semiry, "Long wavelength peristaltic flow in a tubes with an endoscope subjected to magnetic field," Korea-Australia Rheology Journal, vol. 25, pp. 107-118, 2013.

[20] D. Tripathi, S. K. Pandey, and S. Das, "Peristaltic flow of viscoelastic fluid with fractional Maxwell model through a channel," Applied Mathematics and Computation, vol. 215, no. 10, pp. 3645-3654, 2010.

[21] S. Nadeem and N. S. Akbar, "Influence of radially varying MHD on the peristaltic flow in an annulus with heat and mass transfer," Journal of the Taiwan Institute of Chemical Engineers, vol. 41, no. 3, pp. 286-294, 2010.

[22] S. Srinivas, R. Gayathri, and M. Kothandapani, "The influence of slip conditions, wall properties and heat transfer on MHD peristaltic transport," Computer Physics Communications, vol. 180, no. 11, pp. 2115-2122, 2009.

[23] S. Ahmad, M. Yousaf, A. Khan, and G. Zaman, "Magnetohydrodynamic fluid flow and heat transfer over a shrinking sheet under the influence of thermal slip," Heliyon, vol. 4, no. 10, 2018.

[24] Z. Ullah, G. Zaman, and A. Ishak, "Magnetohydrodynamic tangent hyperbolic fluid flow past a stretching sheet," Chinese Journal of Physics, vol. 66, pp. 258-268, 2020.

[25] Z. Ullah and G. Zaman, "Lie group analysis of magnetohydrodynamic tangent hyperbolic fluid flow towards a stretching sheet with slip conditions," Heliyon, vol. 3, no. 11, 2017.

[26] I. Ullah, T. A. Alkanhal, S. Shafie, K. S. Nisar, I. Khan, and O. D. Makinde, "MHD slip flow of Casson fluid along a nonlinear permeable stretching cylinder saturated in a porous medium with chemical reaction, viscous dissipation, and heat generation/absorption," Symmetry, vol. 11, no. 4, p. 531, 2019.

[27] A. S. Alsagri, S. Nasir, T. Gul et al., "MHD thin film flow and thermal analysis of blood with CNTs nanofluid," Coatings, vol. 9, no. 3, p. 175, 2019.

[28] L. A. Lund, D. L. C. Ching, Z. Omar, I. Khan, and K. S. Nisar, "triple local similarity solutions of Darcy-forchheimer magnetohydrodynamic (MHD) flow of micropolar nanofluid over an exponential shrinking surface: stability analysis," Coatings, vol. 9, no. 8, p. 527, 2019.

[29] K. Javid, S. Ud-Din Khan, S. Ud-Din Khan, M. Hassan, A. Khan, and S. A. Alharbi, "Mathematical modeling of magneto-peristaltic propulsion of a viscoelastic fluid through a complex wavy non-uniform channel: an application of hall device in bio-engineering domains," The European Physical Journal Plus, vol. 136182 pages, 2021.

[30] K. Javid, U. F. Alqsair, M. Hassan, M. M. Bhatti, T. Ahmad, and E. Bobescu, "Cilia-Assisted Flow of Viscoelastic Fluid in a Divergent Channel under Porosity Effects," Biomechanics and Modeling In Mechanobiology, 2021.

[31] K. Maqbool, N. Manzoor, S. Poncet, and A. Siddiqui, "inertial flow of viscoelastic second-grade fluid in a ciliated channel under a magnetic field and Darcy's resistance," Applied Science, vol. 11, no. 9, p. 3819, 2021. 
[32] S. Hina, T. Hayat, and A. Slsaedi, "Heat and mass transfer effects on the peristaltic flow of Johnson-Segalman fluid in a curved channel with compliant walls," International Journal of Heat and Mass Transfer, vol. 55, no. 13-14, pp. 3511-3521, 2012.

[33] M. Hameed, A. A. Khan, R. Ellahi, and M. Raza, "Study of magnetic and heat transfer on the peristaltic transport of a fractional second grade fluid in a vertical tube, " Engineering Science and Technology," An International Journal, vol. 18, no. 3, pp. 496-502, 2015. 